\title{
Surgical Hairline Advancement: Patient Candidacy and Best Techniques
}

\author{
Jeffrey S. Epstein, MD I Miami, Florida, USA I jse@drjeffreyepstein.com; Gorana Kuka Epstein, MD I \\ Belgrade, Serbia
}

The growing interest of both patients and surgeons in hairline lowering/forehead reduction surgery (also called surgical hairline advancement, or SHA) is due to the availability of information online, its presentation by different surgeons at ISHRS meetings (e.g., in Prague and the Orlando Live Surgery Workshop), and a Cyberspace Chat discussion in a recent issue of the Forum (2017; 27(5):188-190) concerning a complication due to SHA. Most importantly, patient demand is driven by the recognized advantages of the surgery over hair transplants alone in the appropriate candidate. Just in the past six weeks, the senior author received referrals of three female patients from leading ISHRS surgeons who felt hairline lowering surgery was a better option for these patients. One referral had insufficient graft density and a poor outcome due to a history of frontal fibrosing alopecia.

SHA is unsurpassed in its ability to lower high hairlines by an average of $2.1 \mathrm{~cm}$, however, as much as $5.5 \mathrm{~cm}$ of lowering is possible in exceptional candidates (Figures 1 and 2). This represents the equivalent of transplanting 8,000-10,000 grafts, takes less than two hours to perform, and offers results that are immediately visible. In as little as three months following SHA, the majority of patients undergo hair grafting to further round out the hairline and/or conceal the fine-line hairline scar. The transplants are typically performed by the referring physician.

FIGURE 1. 19-year-old before (A, B), 1 week after (C), and 6 months after (D, E) hairline lowering surgery, where $26 \mathrm{~mm}$ of advancement was achieved.
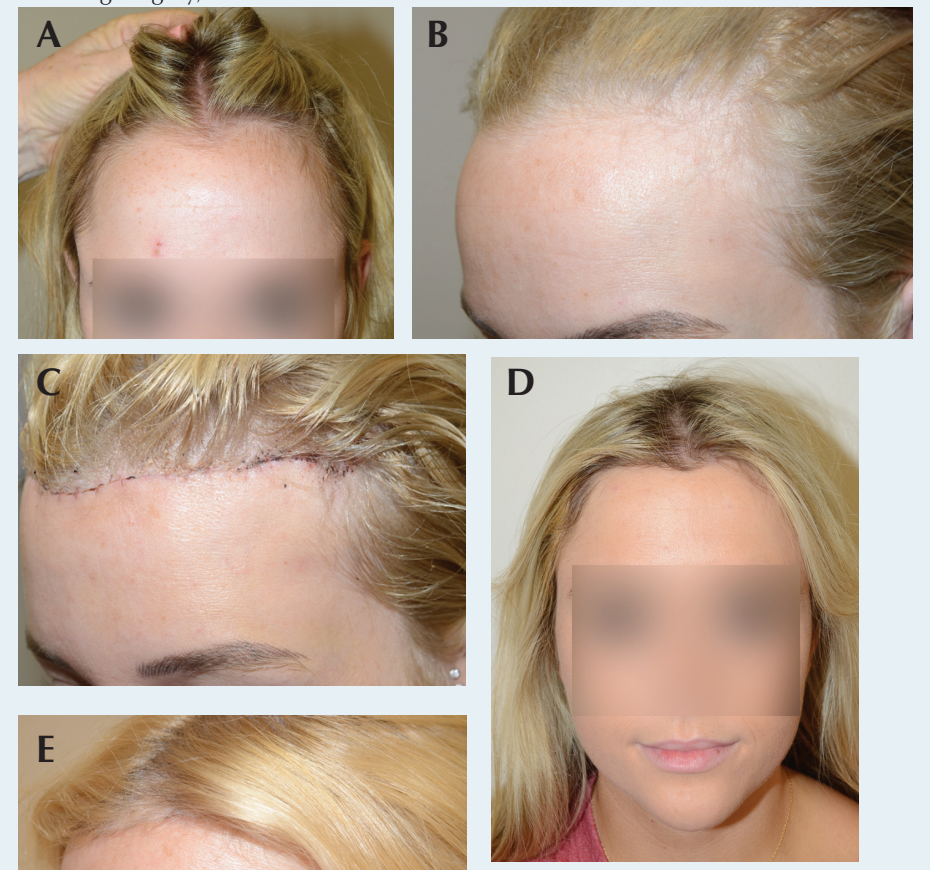

FIGURE 2. African-American patient before (A) and 1 year after (B) hairline lowering surgery.
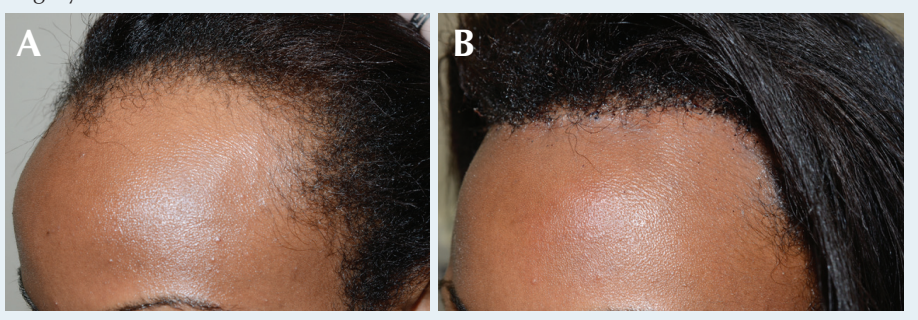

Like any surgical procedure, SHA requires proper execution and determination of candidacy, and when performed properly, SHA has a very low incidence of complications. This surgery was first described in the literature in 1999 by Timothy Marten, a plastic surgeon, as hairline lowering in conjunction with brow lifting. ${ }^{1}$ It has subsequently been written about in conjunction with frontal bone reshaping ${ }^{2}$ and as a stand-alone procedure. ${ }^{3-5}$ It also has appeared in articles in past issues of this journal. ${ }^{6,7}$ Following is a review of the latest modifications in techniques and strategies for achieving the most consistent results, based on performing 89 procedures over the past 9 years, with 37 of them over the past 18 months as patient interest has grown and outcomes have improved due to improvement of techniques.

\section{OVERVIEW}

SHA is a 90-minute procedure typically performed under twilight and ring block anesthesia, but oral sedation is also suitable. Through a trichophytic frontal hairline incision, first described by Mayer and Fleming, the scalp is undermined in the subgaleal plane past the vertex, then galeotomies are performed to further maximize mobility. ${ }^{8}$ The frontal hairline is then secured in its more anterior position, and excess forehead skin excised. With the Kabaker modification to the technique (I was able to observe Dr. Sheldon Kabaker, largely credited as the developer of the procedure, perform several of these surgeries just prior to his retirement last year), it is possible to provide some rounding of the lateral hairline. While possible to combine with a browlift or the taking down of frontal bone prominence in the transitioning male to female patient, SHA is primarily indicated to shorten the high forehead in women. The occasional male patient can be a candidate (Figure 3), however, he must display no frontal hair thinning, and if he is under the age of 40 , he must not have a family history of male pattern hair loss (MPHL).

\section{Patient selection}

When selecting patients for SHA, the following characteristics are essential:

- Good to very good scalp mobility so that the hairline can be manually pushed forward $1.3 \mathrm{~cm}$ or more 
FIGURE 3. Male African-American patient before (A) and 4 months after (B) hairline lowering surgery; performed due to no risk factors for future male pattern hair loss.
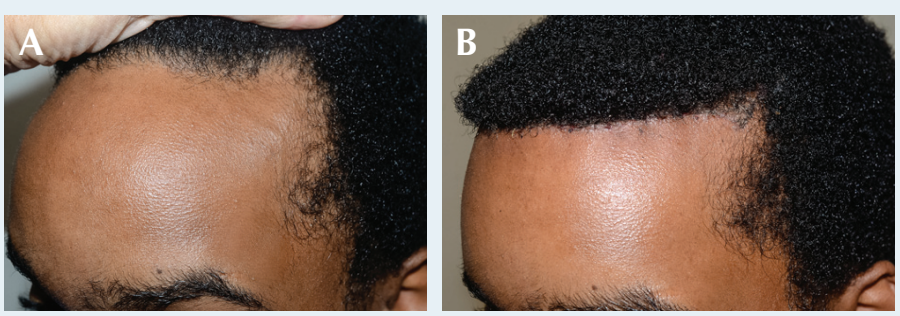

(Figure 4). This forward displacement estimates how far forward the scalp can be advanced, with typically $5-10 \mathrm{~mm}$ of additional advancement achieved from coronal galeotomies. The patient with less mobility can, if highly motivated, undergo balloon tissue expansion over four to six weeks that will allow virtually unlimited advancement (Figure 5).

- A strong frontal hairline without foreseeable risk of thinning.

- Preference for SHA over hair transplantation. SHA is more likely to create a dense, dramatic, virtually instantaneous lowering of the hairline with a fine-line scar in the hairline that may require transplants to help conceal it, whereas hair transplantation alone entails

FIGURE 4. Scalp laxity test determines how far the hairline can be advanced. Before (A), displacement of hairline as far forward as it can be advanced (B), and the marking of the planned location of the lowered hairline (C).
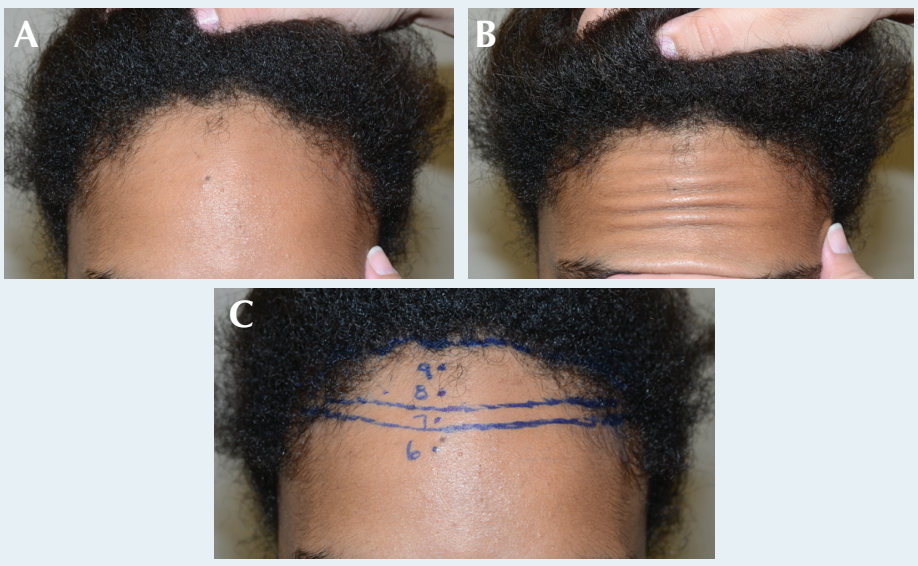

FIGURE 5. Female patient with end-stage frontal fibrosing alopecia who underwent unsuccessful hair grafting 2 years earlier, before (A), after 5 weeks of balloon tissue expansion because of a very immobile scalp (B), and 1 day after hairline lowering surgery (C) where the unaffected frontal hairline was advanced $23 \mathrm{~mm}$ to permit the excision of the prior hair grafts returning her to a more feminine-positioned hairline.
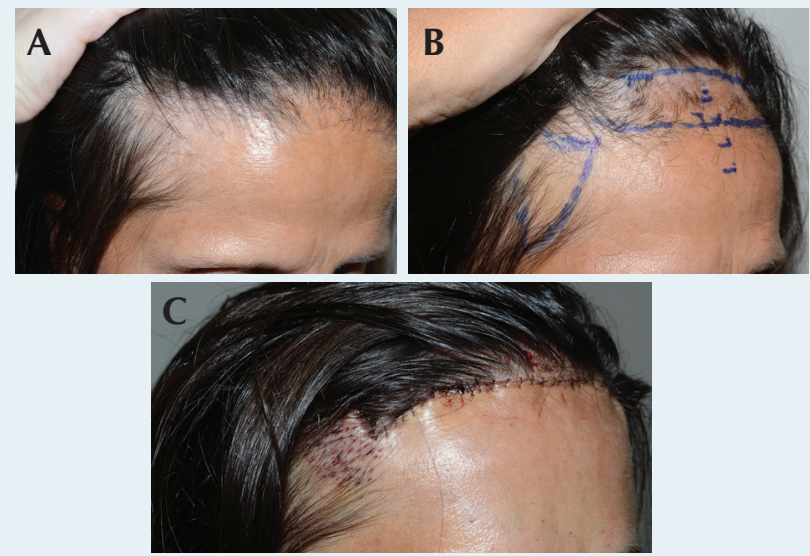

the time for regrowth and may possibly require a second procedure to achieve the desired density.

- No history of prior coronal or temporal browlift, temporal or vertebral artery ligation, or any other condition that compromises blood supply to the frontal scalp. A prior hair transplant is not a contraindication and in fact some patients undergo SHA after being disappointed with the density from a prior transplant.

In addition, the following secondary characteristics are preferred, but not required:

- Hairline hair growth in an anterior direction to allow for hairs to grow through the trichophytic incision.

- The patient desiring primarily vertical shortening rather than horizontal narrowing or rounding of the forehead.

\section{SURGICAL STEPS \\ Design and anesthesia}

The existing hairline is marked out as a somewhat irregular line, just behind any vellus hairs that are too fine to effectively grow through the trichophytic incision. Starting at the midline and proceeding laterally $6-7 \mathrm{~cm}$ from the midline on each side, this line curves gently downwards for $1 \mathrm{~cm}$ to capture the frontal-most finer hairs of the upper temporal/ frontotemporal junction, then takes a right-angle turn and proceeds posteriorly in a horizontal direction for $15-20 \mathrm{~mm}$. A second line is then drawn where the hairline can realistically be advanced, paralleling the shape and irregularity of the initial line (Figure 6).

FIGURE 6. Kabaker modification of incision design, allowing for optimal rounding out of the hairline, before (A) and immediately after (B).
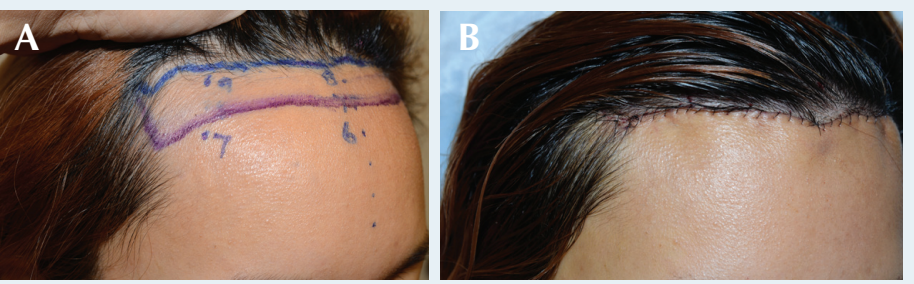

The procedure is usually performed under twilight sedation, or occasionally only oral sedation. Lidocaine anesthesia ( $2 \%$ with epi $1: 100,000)$ of the scalp is achieved with supraorbital and supratrochlear nerve blocks in addition to a ring block around the rest of the scalp. Tumescence with saline significantly reduces bleeding and avoids the need for injecting epinephrine, which can be associated with shock loss.

The hairline incision is made at an acute angle to transect hair follicles, then directly perpendicular through the deeper aspect of the tissue to the periosteum. Dissection is performed with scissors and undermining is done manually in the avascular subgaleal plane to the vertex. This subgaleal dissection is then extended posterior to the vertex another $2-3 \mathrm{~cm}$ with a Deaver retractor optimizing exposure of the typically fibrous tissue that runs between the galea and periosteum in this area. This dissection is similarly extended laterally to the upper parietal and temporal regions to contribute to scalp mobility.

With the scalp optimally undermined, traction is applied to the frontal scalp using three towel clips pulling the scalp 
FIGURE 7. Second of two coronal galeotomies are made with careful incising of the galea. Each galeotomy can provide $3-7 \mathrm{~mm}$ of additional advancement, but must be made carefully to avoid any damage to more superficial vessels.

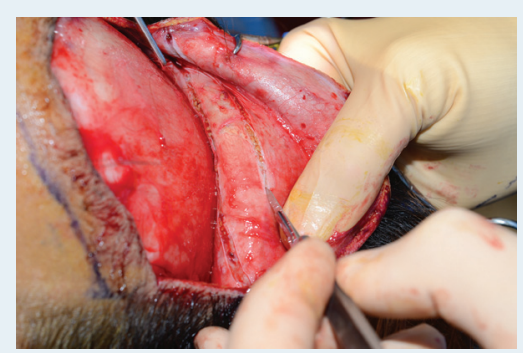

as far anterior as possible, generating as much mechanical creep as possible for 60 seconds. One coronal galeotomy approximately $25 \mathrm{~mm}$ posterior to the leading edge of the frontal scalp and usually a second galeotomy $10-15 \mathrm{~mm}$ behind the first are made with the tip of a \#15 scalpel blade that cuts just through the galea and avoids the vessels that are just superficial to it (Figure 7). After each galeotomy, 60 seconds of traction is applied in the same fashion with on average $2-3 \mathrm{~mm}$, but as much as $7-10 \mathrm{~mm}$ of additional mechanical creep is generated from each galeotomy.

The scalp must be secured in its most anterior position. There are several methods to do this; our preference is using two Endotine ${ }^{\circledR}$ clips because of ease and reliability. The anchor of each Endotine clip is placed into holes drilled into the cranium that are each located approximately $2 \mathrm{~cm}$ lateral to the midline. The small hooks of the clips engage the galea with the scalp pulled as far anterior as possible, securing the hairline at its maximally-advanced position. Over the next six months, although these clips will dissolve, the hairline will not migrate posteriorly.

The forehead skin now overlapped by the advanced frontal scalp is excised. If a browlift is desired, dissection just deep to the frontalis muscle of the forehead is performed to the supraorbital rims to optimize mobility, then the additional skin excised to achieve the desired brow position. The hairline incision is closed with interrupted 3-0 PDS sutures from the scalp galea to the forehead frontalis muscle, then a running 5-0 nylon suture to reapproximate the skin edges in a trichophytic fashion, the forehead skin overlapping the leading edge of the de-epithelialized frontal scalp. The temporal portions of the closure are performed to eliminate a dog-ear deformity.

Antibiotic ointment is applied and a pressure dressing is left in place until the next day, at which time patients are presentable with the hair brushed forward or wearing a cap. Normal hair washing is permitted on the third day, and sutures are removed at one week. By six weeks, hairs grow through the trichophytic incision. Loss of sensation at the frontal scalp, due to cutting the supraorbital and supratrochlear nerve branches at the hairline, resolves typically by $80 \%$ within one year.

\section{HAIR TRANSPLANTS WITH SHA}

Many SHA patients can benefit from transplants, which can be done as soon as three to four months post-surgery. Four hundred to 1,600 grafts can be transplanted to round out the hairline and, if desired and indicated, into and in front of the hairline scar to further reduce its visibility and possibly to lower the hairline a bit more (Figure 8). These grafts can be obtained by FUE or strip technique with no special considerations.
FIGURE 8. Before (A) and 18 months after SHA/14 months after hair grafting (B) to round out the hairline and fill in the upper temporal regions.
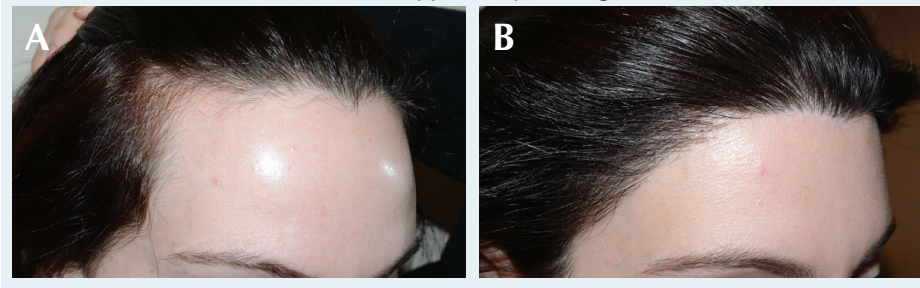

In the occasional patient, grafting is done simultaneously with SHA, with 200-250 grafts placed into each upper temporal region to create a more rounded hairline. These grafts are harvested by the follicular unit excision (FUE) technique from the lower occiput region or are dissected from tissue obtained from the dog-ear repairs along the temples. Because of the lower percentage of regrowth with these simultaneously placed grafts and the difficulty in placing grafts close to the hairline incision, transplanting, if indicated, is best performed three to four months post-op.

\section{RESULTS AND COMPLICATIONS}

The technique described had several modifications made 18 months ago that improved outcomes. We summarize the results on the last 37 patients treated over this time, including 3 males, 2 transgender females, and 32 lifetime females. Four patients underwent balloon tissue expansion due to low scalp mobility. Of the 33 patients not undergoing tissue expansion, the range of hairline advancement was 12$54 \mathrm{~mm}$, with the median $21 \mathrm{~mm}$. Two patients experienced small (less than $1 \mathrm{~cm}$ in diameter) areas of limited hair loss, one over an Endotine clip and the other in the mid-scalp region, both notably on patients with thinner and tighter scalps. Both cases had at least partial regrowth of hair in these small areas, one of whom had approximately 50 grafts placed into that area at the same time as hairline grafting.

Despite online and hearsay reports of significant shock hair loss, no patients experienced shock loss. Two patients had concerns about the hairline scar being visible with their hair pulled back and both were treated with transplants to address this concern. To date, grafting to round out the hairline and/or soften the hairline scar was performed by us on 9 patients, and to our knowledge 4 patients had grafting performed by a local surgeon, with all patients advised prior to having surgery that this may be desired. Because of the up to one year of decreased sensation of the scalp, patients are advised to use caution when blow-drying their hair to avoid thermal injury to the scalp.

\section{THOUGHTS AND TAKE HOME POINTS}

No procedure is without risks, advantages, and disadvantages. Hairline lowering with transplantation alone has its complications, including poor graft regrowth, shock loss to existing hairs, superficial cellulitis/folliculitis, prolonged erythema, and not uncommonly insufficient density that requires a second transplant procedure. SHA also has its risks (which can be significantly minimized) and disadvantages, however, because it has the main advantage of virtually instantaneous lowering of a dense hairline, it should be considered in the appropriate patients. Overall, this is a 


\section{Literature Review}

Nicole E. Rogers, MD, FISHRS I Metairie, Louisiana, USA I nicolerogers11@yahoo.com

\section{New Data on Best Protocol for Platelet-rich Plasma (PRP)}

Hausauer, A.K., and Jones, D.H. Evaluating the efficacy of different platelet-rich plasma regimens for management of androgenetic alopecia: a single-center, blinded, randomized clinical trial. Dermatol Surg. 2018; 44:1191-1200.

A recent publication in the Journal of Dermatologic Surgery (funded by Eclipse Aesthetics LLC) examined two treatment protocols for PRP. In a prospective, randomized, single-blinded trial, 40 patients total (30 men and 10 women) with androgenetic alopecia (AGA) were randomized to receive treatments monthly for the first 3 months, followed by a fourth treatment 3 months later (Group 1), or to receive two treatments 3 months apart (Group 2). Global photographs were taken with the Hair Metrix SM/Canon Rebel T6i; Canfield Scientific Inc. and dermatoscopic photos were taken with the Korean Folliscope 2.8 at baseline, 3 months, and 6 months. The authors demonstrated that there was a similar and statistically significant improvement in hair counts for each group at 6 months, but that only Group 1 had statistically significant increases at 3 months. There were no differences in the increases seen among men and women $(p=.78)$. For hair shaft caliber, statistically significant increases were seen for each group at both 3 and 6 months $(p<.001)$. The authors postulate that it may require a lower concentration PRP-contained growth factors to thicken miniaturized hairs than to transition into anagen phase and promote new growth.

Patients were asked to rate their level of satisfaction as 3 (highly satisfied), 2 (satisfied), 1 (unsatisfied), or 0 (highly unsatisfied). Mean satisfaction across the entire study period was 2.3. Patients in Group 1 were more likely to report the highest scores probably because they perceived earlier results. The authors also used Jeffrey Rapaport's method of fewer subdermal injections, allowing diffusion of the PRP.

Comment: There remains continued debate among hair transplant physicians about the best protocol for PRP to treat hair loss. Some argue that a single injection should be sufficient to augment hair growth. They may justify that the addition of platelet activators or ACell can boost the results with a single session and thus lessen the need for additional treatments. Eclipse $\mathrm{PRP}^{\circledR}$ does not contain any such activators. Rather, it has a proprietary gel plug that removes $99.9 \%$ of erythrocytes and $92 \%$ of leukocytes. The data presented here suggests that the benefits from PRP may be greater (and appreciated earlier) if it is first administered monthly. However, the results may be essentially the same at 6 months. procedure offering a tremendously high level of satisfaction for the appropriate patient.

\section{References}

1. Marten, T.J. Hairline lowering during foreheadplasty. Plast Reconstr Surg. 1999; 103(1):224-236.

2. Cho, S., and H.R. Jin. Feminization of the forehead in a transgender: frontal sinus reshaping combined with brow lift and hairline lowering. Aesth Plast Surg. 2012; 36:1207-1210.

3. Kabaker, S.S., and J.P. Champagne. Hairline lowering. Facial Plast Surgy Clin N Am. 2013; 21:479-486.
4. Guyuron, B., and D.J. Rowe. How to make a long forehead more aesthetic. Aesthetic Surg J. 2008; 28:46-50.

5. Ramirez, A.L., K.H. Ende, and S.S. Kabaker. Correction of the high female hairline. Arch Facial Plast Surg. 2009; 11(2):84-90.

6. Marzola, M. Hairline advancement. Hair Transplant Forum Int'l. 2007; 17(2):67-68.

7. Ramirez, A.L., and S.S. Kabaker. Reducing the female forehead without hair transplantation. Hair Transplant Forum Int'l. 2004; 14(3):93-94.

8. Mayer, T.G., and R.W. Fleming. Aesthetic and Reconstructive Surgery of the Scalp. St. Louis (MO): Mosby-Year Book; 1992. pp. 121-124. 